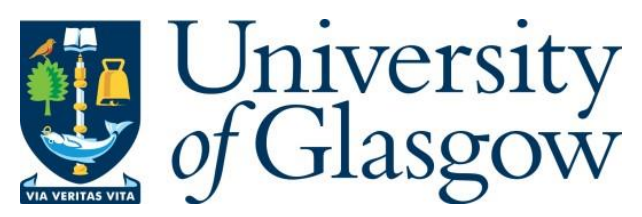

Krämer, S. (2010) How not to defend ontological cheats. Analysis, 70(2), pp. 290-296.

There may be differences between this version and the published version. You are advised to consult the publisher's version if you wish to cite from it.

http://eprints.gla.ac.uk/140562/

Deposited on: 4 May 2017

Enlighten - Research publications by members of the University of Glasgow http://eprints.gla.ac.uk 


\section{How not to defend ontological cheats}

\section{STEPHAN KRÄMER}

- penultimate draft; please cite from original -

Abstract: Jonathan Tallant $\left(2009^{1}\right)$ argues against the view that 'truth requires ground' by trying to show that some theories which violate this principle are theoretically more virtuous than competitors respecting it, which gives us a good reason to reject the principle. I argue that Tallant's argument is unsuccessful.

\section{Introduction}

Many metaphysicians are attracted to a thought I shall call the 'grounding principle': roughly speaking, that for every true proposition, there must be an object that grounds the truth, that makes it true. They sometimes advocate this principle as a way of 'catching ontological cheaters': theorists or theories endorsing claims without endorsing the ontology required by the truth of these claims. Jonathan Tallant tries to show that being a cheat (in the eyes of said metaphysicians) is not so bad: some theories - his examples are versions of actualism and presentism - violating the grounding principle offer significant advantages compared to their 'grounded' competitors. He tries to show this by (among other things) arguing for the following theses:

OP: Rejecting the grounding principle in favour of a weaker alternative allows us to account for the truth of modal and past-tensed claims in a more ontologically parsimonious way, namely without postulating modal or temporal ontology (i.e. non-actual objects and worlds, and non-present objects and times). (Cf. $\S \S 2-3)$

TV: The ontological parsimony gained by those theories violating the grounding principle is

1 In what follows, bare references to page numbers or sections are to this article. 
not counter-balanced by the comparative simplicity and elegance of their competitors since the latter kind of simplicity is not a genuine theoretical virtue, whereas ontological parsimony is. (Cf. §§6-7)

It follows from OP and TV that, other things being equal, the cheat's theories are to be preferred over the grounded alternatives. However, I think that Tallant's arguments for OP and TV are unsuccessful. In $\S 2$ and §3, I present objections against Tallant’s defence of OP; §4 raises doubts concerning his argument for TV.

\section{The grounding principle and Tallant's alternative}

Tallant provides the following explication of the grounding principle, which he ascribes to Bigelow (1988: 126):

$\mathrm{ST}^{2}$ : Necessarily, if $<\mathrm{p}>$ is true, it would be impossible for $<\mathrm{p}>$ to be false unless at least one entity which does not exist were to exist, and at least one entity which exists were not to exist. (422)

This may not be a fortunate explication of the grounding principle for Tallant's purposes, for it is not clear that it has the force he takes it to have when applied to modal propositions. Many philosophers think that possibility and necessity are not themselves contingent matters: if it is necessary (possible) that $p$, then it is necessarily necessary (possible) that $p$. If so, ST is trivially fulfilled for propositions of the form < possibly, $p>$ and $<$ necessarily, $p>-$ if a proposition of this kind is true, it is impossible for it to be false, no matter what - and ST fails to yield a grounding requirement for such propositions. I suggest the following alternative:

GP: Necessarily, for every true proposition (except negative existentials ${ }^{3}$ ), there is something

2 'ST' stands for 'supervenience thesis'; the label is Tallant's. ' $\langle p>$ ' is to be read: the proposition that $p$.

3 By contrast to what Tallant seems to assume (cf. 424), ST does not seem compatible with rejecting the 
which grounds it, which makes it true.

(Spelling out the notion of grounding in such a way as to yield plausible results for modal claims is not trivial, but this seems to be a problem for those wishing to defend the grounding principle. Like Tallant, I shall also call the thing(s) grounding a given truth its 'truthmaker(s)'.)

Tallant proposes that we deny the requirement for an ontological grounding of truths in some cases, in particular, 'the modal and temporal cases' (423). For example, instead of saying, as the proponent of GP does, that the truth of the proposition that there could have been a talking donkey requires that something makes it true - a merely possible talking of a donkey, say - we claim that the truth of this proposition 'requires only that there could have existed the fact of there being a talking donkey' (423, my italics). Similarly, instead of saying, as the proponent of GP does, that the truth of the proposition that Caesar crossed the Rubicon requires that something that makes it true - the past event of Caesar crossing the Rubicon, say - we claim that the truth of this proposition 'requires only that there has existed the fact of Caesar's crossing' (423).

Instead of GP, then, which requires that (almost) every truth be accounted for in terms of the actual existence of a truthmaker, Tallant proposes we adopt a weaker alternative, which allows some of these truths to be accounted for in a different way: in terms of the merely possible or merely past existence of a truthmaker. Here is his attempt at formulating such a principle:

NGC-ST ${ }^{4}$ : a proposition is true if and only if, either: (a) there exists an entity that makes that proposition true; or (b) there does not exist an entity and that makes the proposition true; or (c) there could have existed an entity that would make the proposition true; or (d) there has

grounding principle for negative existentials: $<$ There are no talking donkeys $>$ is true in the actual world. According to ST, any world in which it is false must be a world in which something does not exist which actually does exist. Unless a truthmaker is needed for <there are no talking donkeys>, this seems false: there should be a world containing everything the actual world contains plus (at least) a talking donkey.

4 'NGC' stands for 'no-ground cheating', 'ST', as before, for 'supervenience thesis'. The label is Tallant's. 
existed an entity that makes the proposition true ... (426) ${ }^{5}$

This principle is unacceptable: it yields wildly implausible results and does not capture the view Tallant has in mind. ${ }^{6}$ The strategy of the cheat is supposed to be that of offering different kinds of necessary and sufficient conditions for the truth of different kinds of propositions. The righthand side of the bi-conditional then needs to distinguish these kinds of propositions. As it stands, clause (c), which surely is meant to deal with propositions of the form $<$ possibly, $p>$, is satisfied by various propositions not of this form. For example, <some donkeys talk> satisfies (c) - there could have been something that would make it true - and thus is true according to NGC-ST. Moreover, even when restricted to the pertinent kind of proposition, some of the clauses need to be modified: For example, <possibly, some donkey talks> is supposed to be true because there could have been something that would make true not the proposition itself, as (c) would have it, but the embedded < some donkey talks $>.{ }^{7}$ Clause (b), meant to deal with negative existentials, needs modifying too. As it stands, it is not satisfied by anything, because its first conjunct - 'there does not exist an entity' - is trivially false. ${ }^{8}$ Plausibly, the clause should read: there does not exist an entity which makes the proposition false, i.e. which makes the embedded existential proposition true. (Cf. 424)

A closer look reveals further difficulties. We suggested that the clause for possibility statements should say: there could have been something that makes the embedded proposition true. However, this holds only where the embedded proposition is the kind of proposition that,

5 426, fn. 10 suggests that the dots are meant to indicate that the list might continue should the cheat find good reason to adopt the cheating strategy with respect to further kinds of propositions.

6 Note also that NGC-ST is slightly odd as a replacement for ST: the latter is a strict conditional, whereas the former is a non-modal bi-conditional and thus not a supervenience thesis at all. Presumably, though, the principle is meant to survive modal strengthening and that it offers necessary and sufficient conditions for a proposition's being true is hardly an objection.

7 If, say, 'there is a King of France' expresses the same proposition at different times, with that proposition changing its truth value over time, then an analogous problem affects clause (d). - Similar problems arise for Tallant's principles prefixed 'NGC in the modal / temporal case' on p. 423.

8 Since none of the other clauses are conjunctive, one might conjecture that the 'and' is not meant to be there; the result of deleting it, however, would be the negation of clause (a), and NGC-ST would say that every proposition is true. 
if true, is made true by something according to the cheat. It does not hold, for example, if the embedded proposition is a negative existential: <possibly, there are no human beings> is not true because there could have been a truthmaker for <there are no human beings $>$ but because there need not have been a truthmaker for the existential proposition embedded in the latter proposition. We can avoid this problem by adopting 'possibly, the embedded proposition is true' as the clause for possibility propositions, because this clause is neutral with regard to how the truth of the embedded proposition would be accounted for. My suggestion for how to go about repairing NGC-ST, then, is this: Call any proposition for which the cheat wants to require a truthmaker a TM-proposition. Let $\operatorname{poss}(x)$ and $\operatorname{past}(x)$ be functions from a proposition $x$ to the proposition of the form <possibly, $p>$ and $<$ it was the case that $p>$, respectively, where $x=\langle p>$. The bi-conditional then reads:

NGC-ST*: a proposition $x$ is true iff: (a) $x$ is a TM-proposition and there is something that makes $x$ true; or (b) $x$ is a negative existential and nothing makes the embedded existential proposition true; or (c) for some proposition $y, x=\operatorname{poss}(y)$ and $y$ could have been true; or (d) for some proposition $y, x=\operatorname{past}(y)$ and $y$ was true ${ }^{9}$; or $\ldots$

The dots indicate that the cheat may want to add further disjuncts, as I think he definitely should: For example, without extra clauses for conjunctive propositions, NGC-ST* requires truthmakers for propositions like <there are no talking donkeys and there are no talking monkeys $>$ which seems implausible given that the conjuncts don't require truthmakers. As it stands, NGC-ST* also does not seem to deal properly with negative statements about the past or what is possible. (Both problems also affect Tallant's NGC-ST.) All this goes to show that there remains work for the cheat to do. Tallant has not offered a plausible substitute for GP that does the job he wants.

9 This assumes that the cheat's propositions can change their truth value over time, so that $<$ there is a King of France $>$, for example, was once true, but is now false. 


\section{Avoiding modal and temporal ontology}

There is another area where work remains to be done. Tallant wants to defend denying GP for 'the modal and the temporal cases' (423). In order to succeed in this, he has to tell us how we are to account for all the modal and temporal truths without invoking unwanted ontology. He does tell us how we are to do this for the proposition that there could have been a talking donkey and the proposition that Caesar crossed the Rubicon. The strategy he uses generalises straightforwardly to all truths of the form $\langle$ possibly, $p>$ and $<$ it was the case that $p>$, respectively - NGC-ST* in effect incorporates such a generalisation. Tallant appears to tacitly assume that either, these are all the relevant truths, or, that his strategy for handling these cases generalises equally straightforwardly to all the other cases.

In fact, both disjuncts of this assumption are false. The examples Tallant discusses do not (at least not explicitly) quantify over past objects or mere possibilia; they merely involve temporal and modal operators, which the proponent of GP reduces in terms of such quantification. Tallant's strategy here is to refuse to make this reduction. This strategy does not apply in cases where the propositions whose truth is to be explained already quantify over temporal or modal ontology. Prima facie, there are true propositions of this kind. Suppose there were exactly $n$ Emperors of Rome. (The example is Williamson's (2002: 245).) How do we account for the truth of this proposition without invoking past objects? Tallant's strategy could only be applied to this example if the logical form of the statement was 'it was the case that: there are exactly $n$ Emperors of Rome'. But it isn't, its logical form is: 'for $n$ objects $x$ : it was the case that $x$ is Emperor of Rome'. Its truth therefore seems to require the existence of past objects. It has also been argued that many intuitively correct modal propositions cannot be expressed without quantifying over possibilia or possible worlds. Potential examples include: there could have been other things than there actually are (quantification over possibilia); there 
are three ways I could win this chess game (quantification over possible worlds). ${ }^{10}$

I conclude that Tallant's defence of $\mathrm{OP}$ - the claim that violating the grounding principle lets us avoid a commitment to past objects and merely possible worlds - is incomplete: there are various kinds of intuitively true propositions apparently carrying such commitments to which the cheating strategy does not apply.

\section{Simple theories and simple worlds}

We now turn to TV, the second of Tallant's claims I mentioned in the introduction. Assume for now that we have a cheating strategy which actually allows us to dispense with modal and temporal ontology. Tallant concedes that this benefit is not to be gained without paying a price: the cheat's theory will be significantly more complicated than that of his opponent (call him the 'grounder'). (Cf. 426) One might therefore wonder why one should prefer cheating to grounding: might we not simply have a stand-off, where the theories mutual advantages and disadvantages cancel each other out? Tallant thinks not. He claims that the world postulated by the cheat is much simpler than that postulated by the grounder. (Cf. 426f) Drawing on a point of Joseph Melia's (2000: 473f), he then concludes that as far as simplicity is concerned, the cheat's theory is preferable, since the kind of simplicity we ought to value in theory choice is simplicity of the world postulated by the theory. But why should we accept that the cheat's world is simpler than the grounder's?

Since Tallant appeals to Melia's paper, let us have a look at the pertinent passage. Melia compares two theories $\mathrm{T}_{1}$ and $\mathrm{T}_{2}$ describing spatial relations between physical objects. In order to do this, $\mathrm{T}_{1}$ uses an infinite stock of primitive distance predicates ' $x$ is-1-metre-from $y$ ', ' $x$ is2-metres-from $y$ ', etc., whereas $\mathrm{T}_{2}$ uses the three-place predicate ' $x$ is $r$ metres from $y$ ', where $r$ ranges over the real numbers. While $T_{2}$ is ontologically less parsimonious than $T_{1}$, there is a

10 On these issues, see e.g. Melia 2003: ch. 2, Lewis 1986: $13 \mathrm{ff}$. 
good sense in which $T_{2}$ is a much simpler theory than $T_{1}$. But, argues Melia, this simplicity attaches merely to 'the formulation of the theory itself' (2000: 473); it does not translate into simplicity of the world postulated by the theory. The argument crucially relies on the premise that even according to $T_{2}$, the fact of $a$ 's being 2 metres from $b$ does not consist in a three-place relation obtaining between $a, b$, and the number 2 . Therefore, 'for all $\mathrm{T}_{2}$ says, it postulates no fewer fundamental distance relations than $\mathrm{T}_{1}$ and so there is no reason to suppose that the kind of world postulated by $\mathrm{T}_{2}$ is simpler than $\mathrm{T}_{1}$ ' (2000: 473). All that the quantification over the real numbers allows $T_{2}$ to do is generate predicates expressing the fundamental distance relations in a particularly simple and elegant way. As one might also put it, $\mathrm{T}_{1}$ and $\mathrm{T}_{2}$ agree about the nature of distance-facts, but only $\mathrm{T}_{2}$ invokes mathematical objects to describe those facts.

The situation of the grounder and the cheat is very different: the grounder does not appeal to modal and temporal ontology merely to generate the linguistic resources for describing a reality he and the cheat are in agreement about. The grounder thinks that the fact of Caesar having crossed the Rubicon consists in the existence of certain past objects; the cheat denies this. Grounder and cheat disagree about the nature of modal and temporal facts; they do not simply employ different methods for describing those facts. So Melia's argument for disregarding a certain kind of complexity when choosing which theory to accept does not extend to the case Tallant is concerned with; Tallant owes us an argument for disregarding the complexity of the cheat's theory.

To strengthen my point, I'll try to make a prima facie case that we should not do that. The grounder's world includes past objects and times, as well as merely possible objects and worlds. A world without those kinds of objects and otherwise like that of the grounder would certainly be simpler. But while the cheat's world does not include those kinds of objects, it is not otherwise like that of the grounder. The grounder's world (or perhaps: its fundamental level) 
can be exhaustively described by saying what kinds of things it contains. The cheat's cannot. Let $F_{G}$ be the set of propositions the grounder accepts as fundamental truths about the world. Now remove those members from $F_{G}$ that postulate modal and temporal ontology; call the result $F^{*}$. The cheat cannot accept $F^{*}$ as a complete account of how the world is at the fundamental level. He has to say that $F^{*}$ leaves out important facts about the world, those which concern what kinds of things it has, or could have, contained. In a good sense of 'complex', I submit, he thinks that the world is more complex than $F^{*}$ has it. This sense of 'complex' is relevant to theory choice: other things being equal, a theory on which the world is as $F^{*}$ has it is preferable to both the grounder's and the cheat's theory. ${ }^{11}$ So while the cheat's world is simpler than the grounder's in one respect, namely that of ontological complexity, it is more complex in another, which we might call propositional, or ideological, complexity. Both kinds of complexity are relevant to theory choice, and it is at best an open question whether cheat or grounder can claim a comparative advantage in terms of overall simplicity. Tallant has not established that cheating delivers the benefits he claims for it. ${ }^{12}$

University of Leeds

Leeds LS2 9JD, UK

phsk@leeds.ac.uk

\section{References}

Bigelow, J. 1988. The Reality of Numbers: A Physicalist's Philosophy of Mathematics. Oxford: OUP.

Lewis, D. 1986. The Plurality of Worlds. Oxford: Blackwell.

Melia, J. 2000. Weaseling away the indispensability argument. Mind 109: 455-79.

11 Of course, it is doubtful that other things are equal, but that is irrelevant to the present point. 12 I would like to thank Duncan Watson for helpful comments. 
Melia, J. 2003. Modality. Chesham: Acumen.

Tallant, J. 2009. Ontological cheats might just prosper. Analysis 69: 422-30.

Williamson, T. 2002. Necessary existents. In Logic, Thought and Language, ed. A. O’Hear, 233-51. Cambridge: CUP. 